Research Article

Open Access

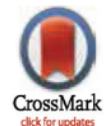

${ }^{1}$ The Oke- Ogun Polytechnic, Saki Oyo State Nigeria, Department of Science Laboratory Technology, Microbiology option.

${ }^{2}$ Ladoke Akintola University of Technology, Ogbomosho, P.M.B 4000, Department of

Pure \& Applied Biology.

${ }^{3}$ Department of Microbiology and Botany,

University of Ibadan, Ibadan, Nigeria.

${ }^{4}$ Department of Zoology, Parasitology Unit,

University of Ibadan, Nigeria.

${ }^{5}$ Cellular Parasitology Unit, Department of

Zoology, University of Ibadan, Nigeria.

${ }^{6}$ Department Food Science Technology, the Oke-Ogun Polytechnic, Saki.

${ }^{7}$ Lead City University, Ibadan, Faculty of Medical Science, Chemical Science

(Biochemistry Unit).

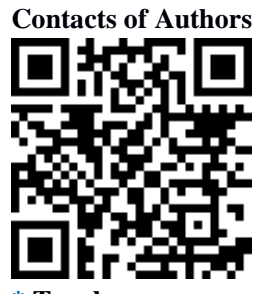

* To whom correspondence should be addressed: Adeoti Olatunde Micheal

Citation: Adeoti Olatunde Micheal, Oni Abosede Catherine, Adeoye Kafilat Adenike, Adeoti Oluwole Adeola, Adeoye Basirat Adedamola and Adesina David Ademola (2020). Predictive comparative antibiotic resistance profiles of rhizobacteria genes using CARD: a bioinformatics approach. Highlights in BioScience Volume 3. Article ID 20223. dio:10.36462/H.BioSci.20223

Received: June 23, 2020

Accepted: September 7, 2020

Published: September 26, 2020

Copyright: () 2020 Micheal et al. This is an open access article distributed under the terms of the Creative Commons Attribution License, which permits unrestricted use, distribution, and reproduction in any medium, provided the original author and source are credited.

Data Availability Statement: All relevant data are within the paper and supplementary materials

Funding: The authors have no support or funding to report.

Competing interests: The authors declare that they have no competing interests.

\section{Predictive comparative antibiotic resistance (AMR) profiles of rhizobacteria genes using CARD: a bioinformatics approach}

\author{
Adeoti Olatunde Micheal ${ }^{1,2,5}$, Oni Abosede Catherine ${ }^{1}$, Adeoye Kafilat \\ Adenike $^{1,4}$, Adeoti Oluwole Adeola ${ }^{6}$, Adeoye Basirat Adedamola ${ }^{7}$ and \\ Adesina David Ademola ${ }^{1,3}$
}

\section{Abstract \\ Members of the Plant Growth Promoting Rhizobacteria (PGPR)} have been severally implicated as excellent growth enhancers, yield promoters as well as bio-fertilizers. A study on antibiotics surveillance of PGPR is urgently needed as caution towards its continued usage in Bioscience and Agro-allied. Antimicrobial resistance has become a great concern in agriculture and public health. The detection and characterization of antimicrobial resistance move from targeted culture and enzyme-based reaction to high-throughput metagenomics; acceptable resources for the analysis of large-scale information area unit as an expected rescue. The excellent bioinformatics tool newly curated for Antibiotic Resistance information (CARD; https://card.mcmaster.ca) could be a curated hub and resource-providing-referenced server for deoxyribonucleic acid and protein sequences as well as detection models on the molecular radar for antimicrobial resistance. This study employed CARD as pathogenomics repertoires for high-quality reference information on retrieving antibiotics resistance information on twentytwo carefully-selected members of Rhizobacter from NCBI. NCBI and CARD on-line platform were employed in polishing of antiobitics resistance info of selected PGPR genera such as Leguminosarum, Azotobacter, Azospirillum, Erwinia, Mesorhizobium, Flavobacterium Paenibacillus Polymyxa, Bacilli mycoides, B. subtilis, and Burkholderia pseudomallei among others. The data generated showed evidence that these rhizobacteria could be resistant to certain drug classes under a different Antimicrobial Resistance (AMR) Gene families using different phyto-pathogenic genes (ARO terms) using different resistance mechanisms. This distinctive platform provides bioinformatics tool that bridges antibiotic resistance considerations, which could be a fallback for policies in healthcare, agriculture and the environment.

Keywords: Plant Growth Promoting Rhizobacteria (PGPR), Comprehensive Antibiotic Resistance Database (CARD), Antimicrobial Resistance (AMR), Phyto-pathogenic, Metagenomics, Bio-fertilizers 


\section{Introduction}

Rhizobacteria are a group of agronomic bacteria that form a mutualistic symbiotic association, which is beneficial to both parties. Members of rhizobacteria are an essential group of microorganisms used as bio-fertilizer [1, $2,3]$ and plant growth promoters, which are often referred to as PGPRs $[4,5,6]$. PGPR enhance plant growth by adding nutrients which act as inoculants during bio remediation, phytostimulation and biological control are classified based on their essential roles in the plant they inhabit $[7,8,9]$. The most common of PGPR species which are present in the rhizosphere are members of the genus Azospirillum $\quad[10,11], \quad$ Bacillus $\quad$ spp. and Pseudomonas spp [12, 13, 14, 15]. The increasing reliance on plants as major sources of pharmaceuticals, cosmetics, and fragrance flavours to meet with the increasing rise in the world population hence the urgent need to investigate its potential antibiotic potentials if barriers of its nonpathogenicity are trespassed $[16,17,18]$.

Recent advancements in pathogenomics have advanced the phenomenon of antimicrobial resistance has gained benchmarking astute as a world number one public health threat $[19,20]$. Therefore, efforts should be dissipated toward the characterization of antimicrobial resistance with increasing attention at the international level and proven to global acceptance in recent United Nations radars. The enzyme based polymerase chain reaction (PCR) characterizes and effectively serve as indicator of pathogenic microorganisms such as Escherichia coli, Salmonella. Non-pathogenicity is associated with Antimicrobial Resistance whereas culture- and PCR-based may have provided the necessary sigh into the prevalence of resistance. These techniques had thus enhance our ability to review both the evolution and ecology of antimicrobial resistance among the entire microbiota population level [21, 22].

There are several web servers exist nowadays that completely identify ontological AMR genes. These customized in silico resources are primarily designed for screening of one ordination or many assembled contigs of Antibiotic Resistance metaphysics (ARO) developed by excellent antibiotic resistance information [21], which is a notable improvement in AMR bio curation. Such an annotational classification is incredibly useful for useful description. The CARD includes bioinformatics tools that modify the identification of antibiotic resistance genes from whole- or partial-genome sequence information together with unannotated raw sequence assembly contigs [23].

Over the ages, because of the evolutionary and ecological drift, there are many bacteria of different genera that have migrated beyond the coast of their ecological niches in an attempt to seek new abode and survival. Some of these organisms include members of Pseudomonas, Serratia, and Escherichia, which have variously acquired resistance complexity along the wheel of change. With this in mind, PGPRs are beneficial today because of their various roles in agriculture and food security, hence the need for predictive genomic resistance profile as a tool for antibiotic resistance for future age.

The present study is an in silico surveillance attempt to predict AMR molecules among farmers friendly PGPR bacteria, although presently environmental opportunistic isolates. PGPR influence plant growth in two different ways: direct and indirect. The direct promotion of plant growth by PGBR is by the synthesis of phyto-hormones, thereby facilitating the uptake of certain nutrients from the environment. Indirect plant growth promotion occurs when PGBR lessen or the risk of phytopathogenic organisms, making them serve as antibiotic by producing bio-control synthesis. We aimed at using bioinformatics tool to predict the antibiotic resistance genes in PGBR.

\section{Materials and Methods}

Retrieval of complete genomes from NCBI: To analyze antimicrobial resistance genes, twenty-two complete genomes of selected Plant Growth Promoting Rhizobacteria (PGPR) complete sequences were randomly retrieved from NCBI database. The retrieved sequences were in FASTA format, which was copied from the National Center for Biotechnology Information NCBI) website; https://www. ncbi.nlm.nih.gov as well as their accession numbers of PGPR.

Analyzing nucleotide sequence on CARD: Nucleotide sequences of twenty two members of PGPR was imported into the CARD analyzing software from genbank using custom software developed specifically for the retention of all annotations, NCBI accession numbers and taxonomy identification (ID) numbers of the PGPR. The importation of these follows a process in which sequences were first acquired from genbank in FASTA format (https://www.ncbi.nlm.nih.gov/) and then loaded into the CARD's Chado database [24]. By convention, CARD uses only the subset of the available NCBI that is relevant to antibiotic-resistant bacteria. Individual Antibiotic Resistance Ontology (ARO) terms in the CARD have been associated with specific computational tools and models $[25,26,14]$.

\section{Results}

Chart representation of RGI results for AMR genes and AMR family as retrieved by CARD on 22 selected PGPR (Figure 1). 


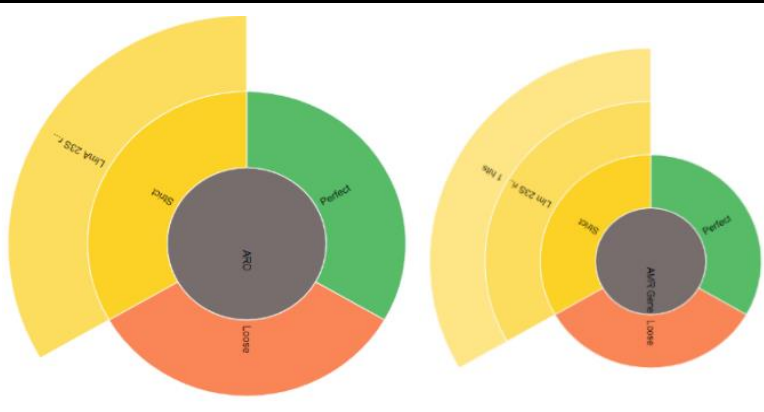

a: AMR gene family of CP0422721.1
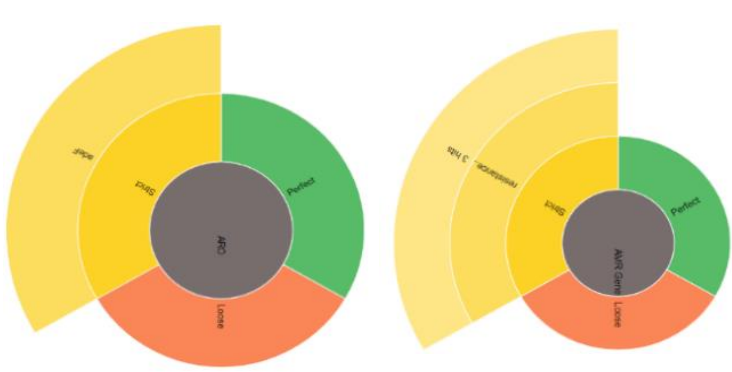

d: AMR gene family of CP019171.1

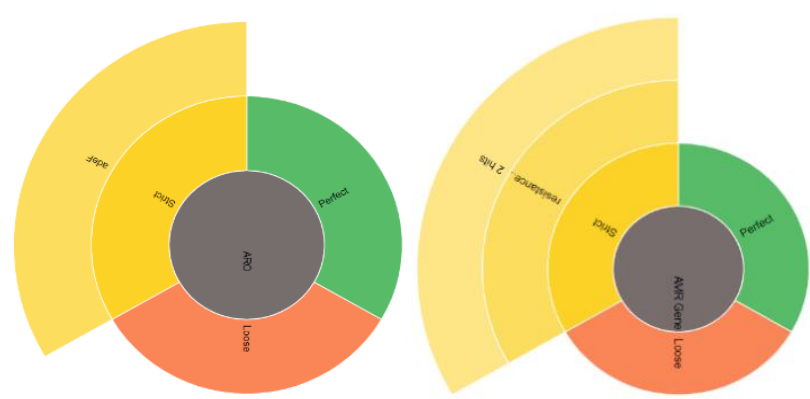

g: AMR gene family for CP033430.1

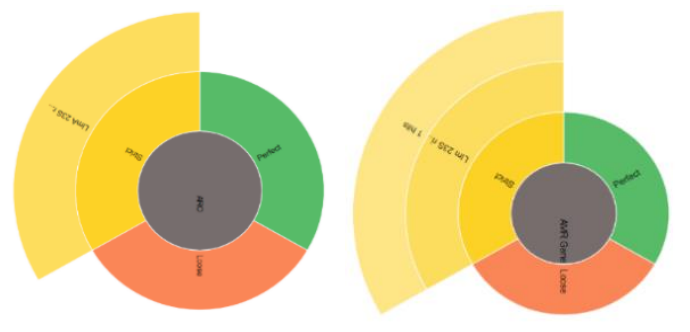

b: AMR gene family of CP021965.1

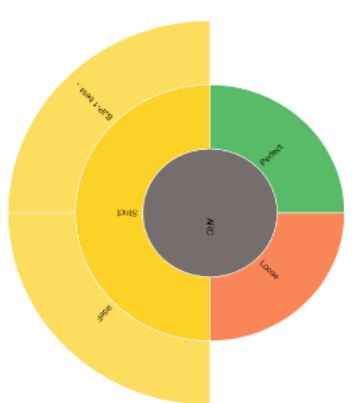

e: AMR gene family for CP010313.1

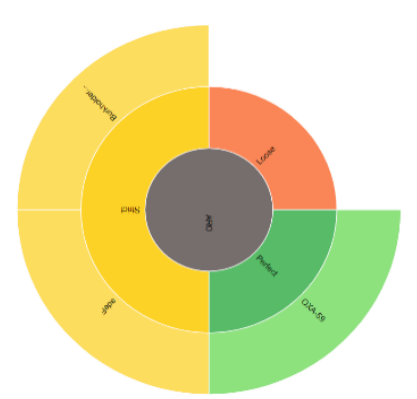

h: AMR gene family of BX571966.1

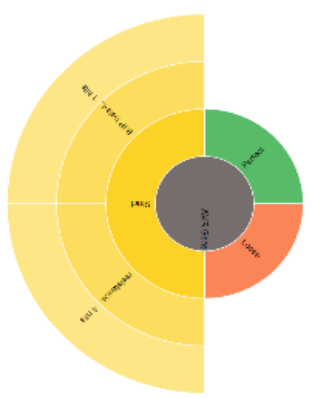

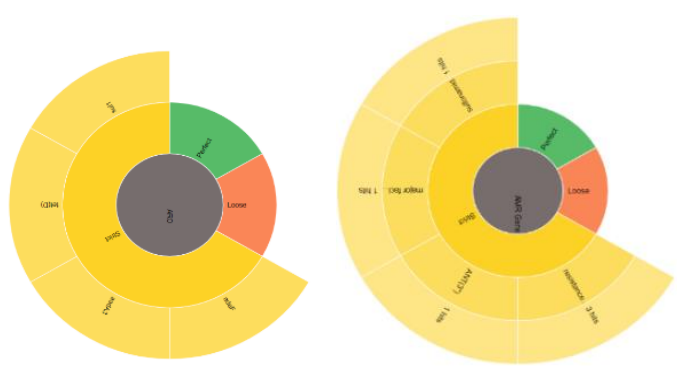

C: AMR Gene family of CP045291.1
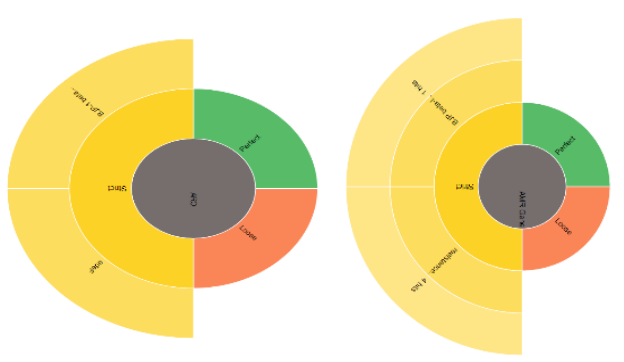

f: AMR gene family of CP029603.1

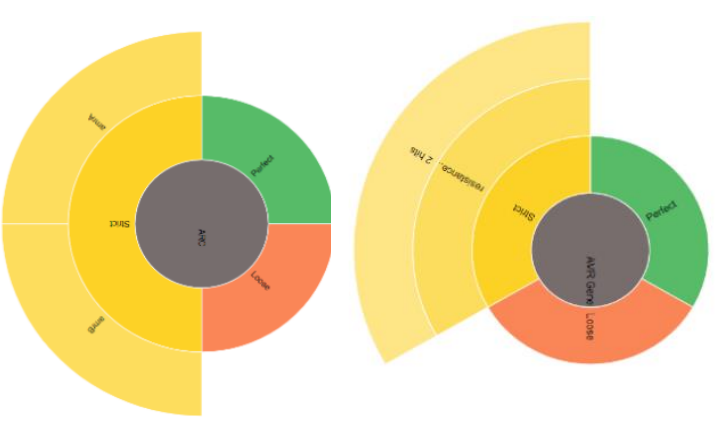

i: AMR gene family of CP040552.1

Figure 1. Continued 


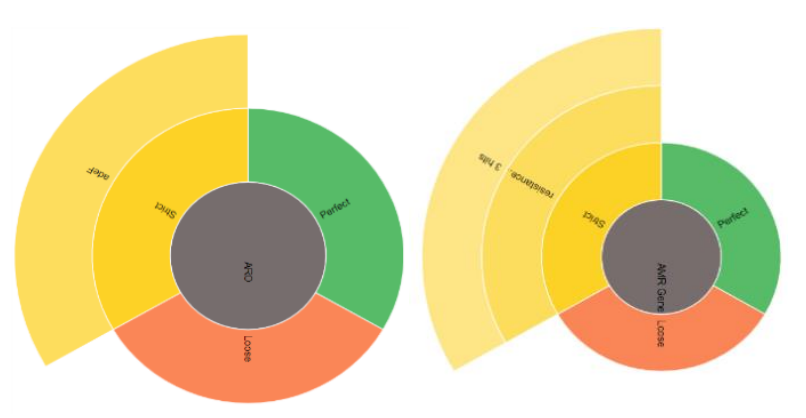

j: AMR gene family of CP018228.1

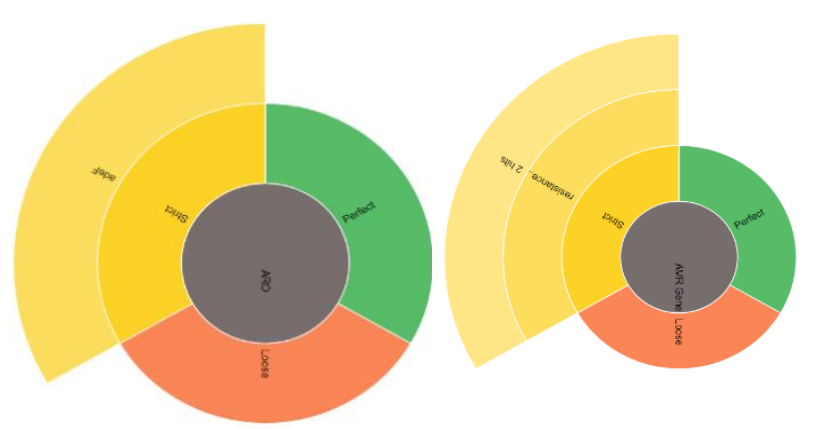

m: AMR gene family for CP000133.1
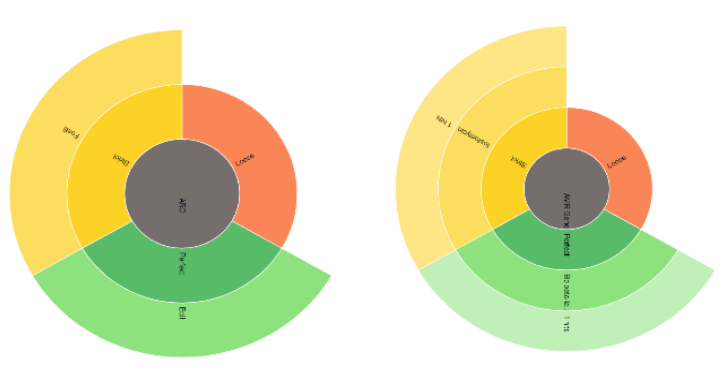

p: AMR gene family for CPO.107209.1

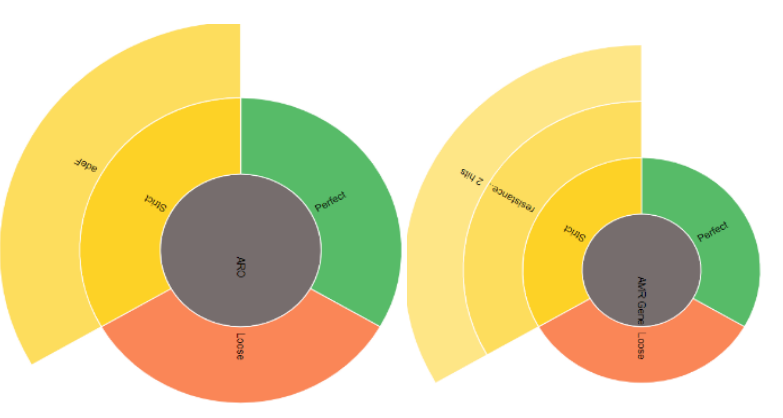

k: AMR gene family for CP004015.1

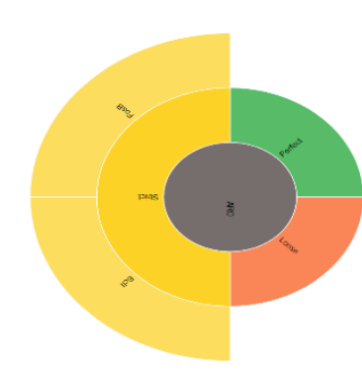

n: AMR gene family for CP037992.1

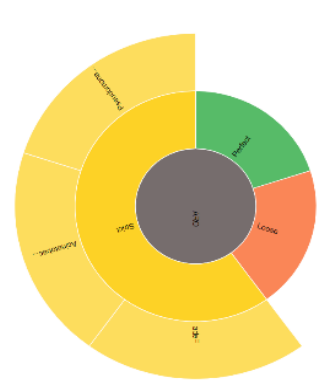

q: AMR gene family for CP037992.1
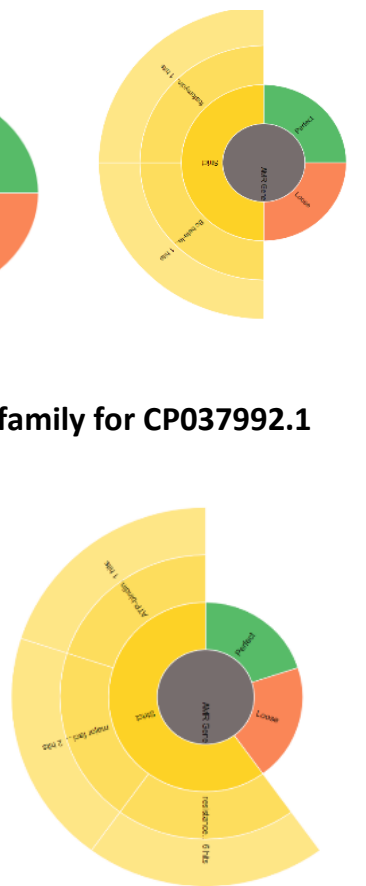

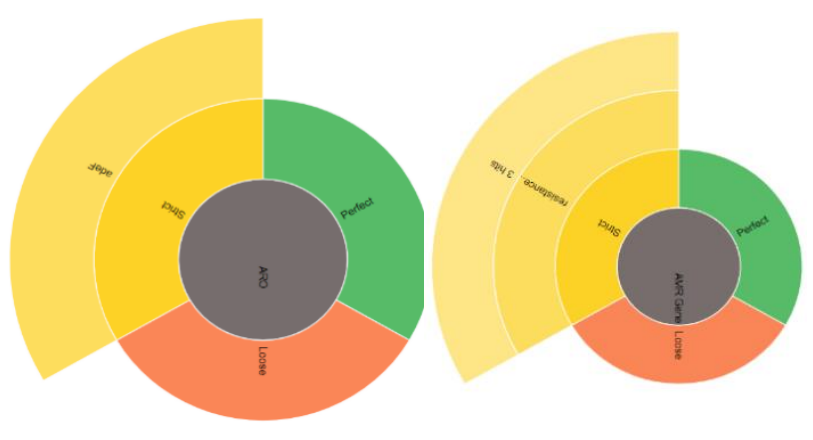

I: AMR gene family for CP18228.1
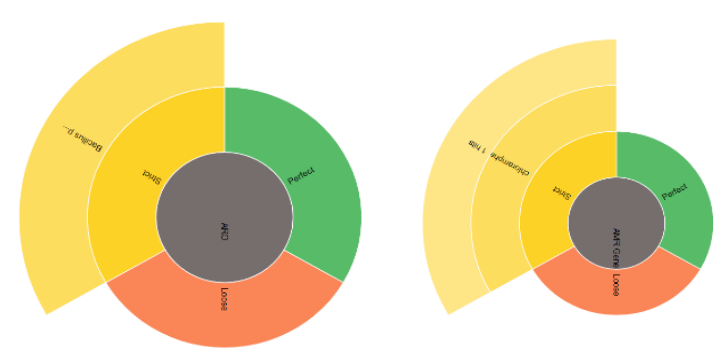

o: AMR gene family for CP027116.1
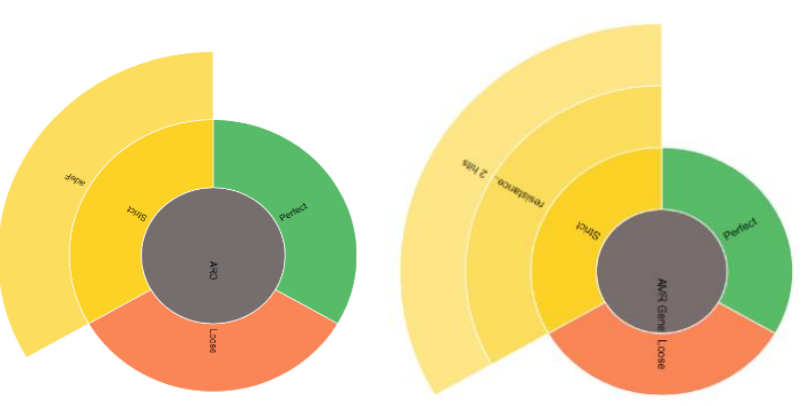

r: AMR gene family for CP025542.1

Figure 1. Continued 

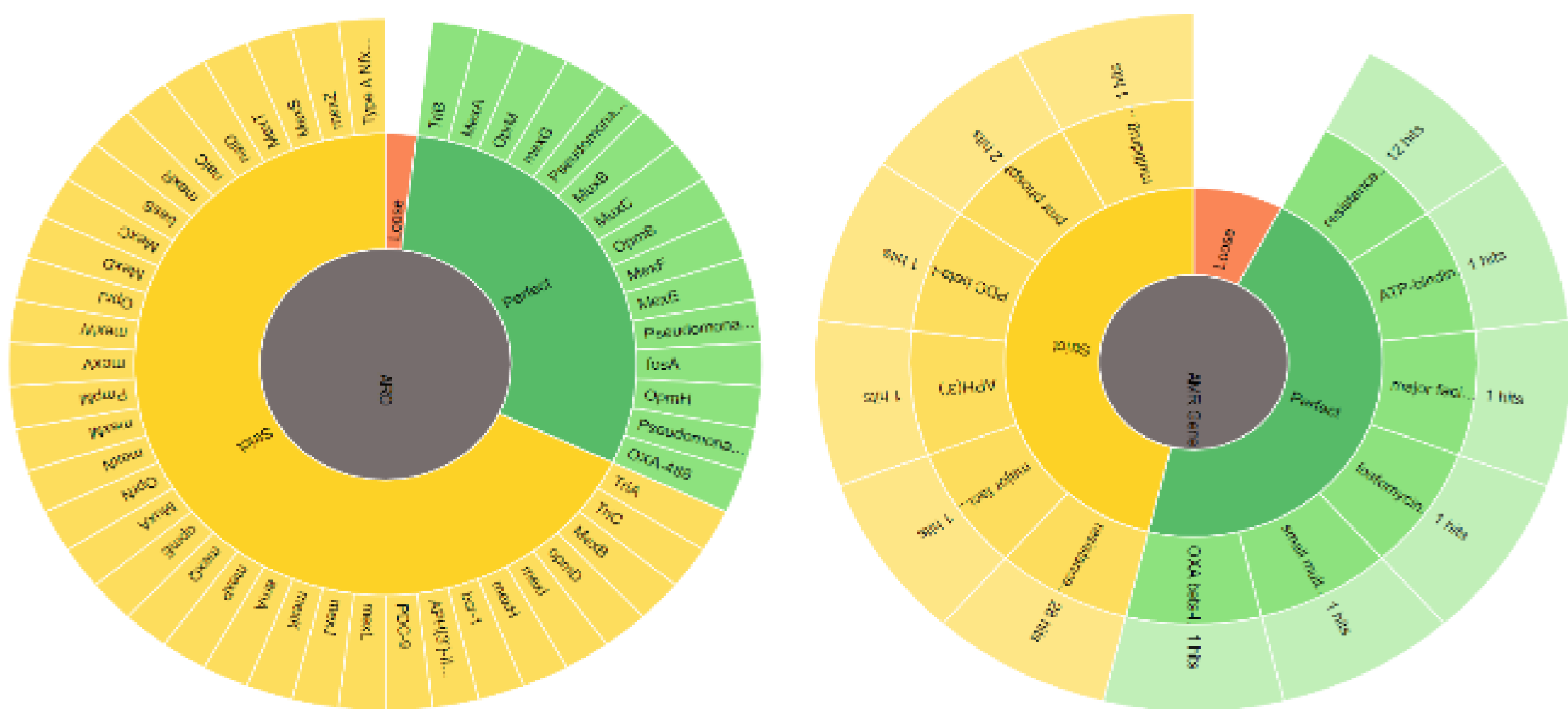

s: AMR gene familv for AP025149.2

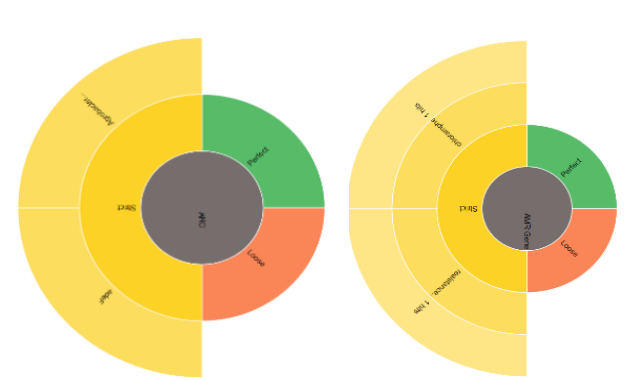

t: AMR gene familv for CP000438.1

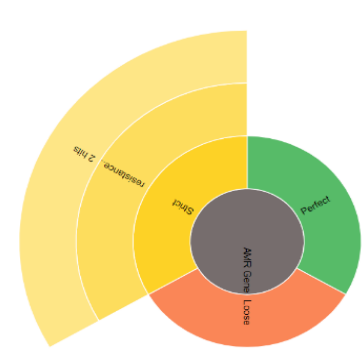

U: AMR gene familv for CP019702.2

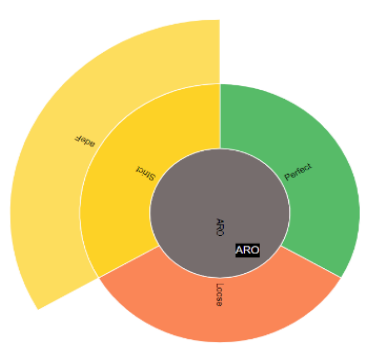

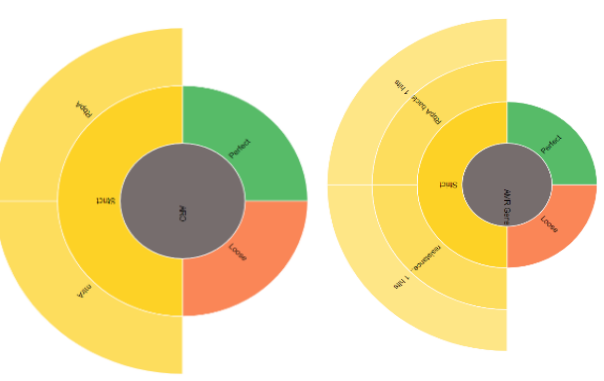

v: AMR gene familv for CP034395.1

Figure 1. ARO classification tags for the selected twenty-two PGRD bacteria a-v: The assemly contigs retains the RGI into perfect (green), strict (flexible )and loose( spurious partial) resistomes. 
Table 1. Prevalence of AMR gene family among PGPRs.

\begin{tabular}{l|llll}
\hline & \multirow{2}{*}{ Accession No } & \multicolumn{3}{c}{ Prevalence of AMR genes } \\
\cline { 3 - 5 } & & Perfect $(\boldsymbol{\%})$ & Strict $(\boldsymbol{\%})$ & Loose $(\boldsymbol{\%})$ \\
\hline $\mathbf{1}$ & CP 042272.1 & 33 & 34 & 33 \\
$\mathbf{2}$ & CP021965.1 & 30 & 40 & 30 \\
$\mathbf{3}$ & CP045291.1 & 20 & 60 & 20 \\
$\mathbf{4}$ & CP019171.1 & 35 & 35 & 30 \\
$\mathbf{5}$ & CP010313.1 & 25 & 50 & 25 \\
$\mathbf{6}$ & CP029603.1 & 25 & 50 & 25 \\
$\mathbf{7}$ & CP033430.1 & 30 & 40 & 30 \\
$\mathbf{8}$ & BX571966.1 & 25 & 50 & 25 \\
$\mathbf{9}$ & CP040552.1 & 25 & 50 & 25 \\
$\mathbf{1 0}$ & CP018228.1 & 30 & 40 & 30 \\
$\mathbf{1 1}$ & CP004015.1 & 44 & 28 & 28 \\
$\mathbf{1 2}$ & CP018228.1 & 23 & 38 & 29 \\
$\mathbf{1 3}$ & CP000133.1 & 35 & 30 & 35 \\
$\mathbf{1 4}$ & CP037992.1 & 25 & 50 & 25 \\
$\mathbf{1 5}$ & CP027116.1 & 33 & 43 & 24 \\
$\mathbf{1 6}$ & AP007209.1 & 40 & 25 & 35 \\
$\mathbf{1 7}$ & CP037992.1 & 23 & 54 & 23 \\
$\mathbf{1 8}$ & CP025542.1 & 13 & 74 & 14 \\
$\mathbf{1 9}$ & CP025149.2 & 38 & 38 & 24 \\
$\mathbf{2 0}$ & CP000438.1 & 48 & 48 & 04 \\
$\mathbf{2 1}$ & CP019702.2 & 25 & 50 & 25 \\
$\mathbf{2 2}$ & CP034395.1 & 34 & 44 & 22 \\
\hline & & & &
\end{tabular}

\section{Discussion}

This study employed CARD ARO and AMR resistomes classification in agreement with earlier studies. The RGI genes in CARD predict resistomes for genomic and metagenomics data for gene mutations from NCBI retrieved nucleotide sequences by using a combination of open reading frame prediction [29, 30]. In consonance with earlier studies, in this study, the perfect algorithm predicted AMR proteins with exact homology $(100 \%)$ with a query on the CARD reference sequences. In the same vein, members of PGPR on CARD algorithm showed strict genes under AMR/ARO curation because strict RGI genes are more flexible by allowing flexible variation from the genome reference sequence within the curated BLAST cut-off, which is useful for detection of previously unreported variants of antibiotics target changes through altered sequences [31].

The loose algorithm under CARD was an indication of resistance genes, which work outside the detection of target, which could enable the detection of new, emerging risks and more distant homologs of antibiotics resistance genes. The loose portends a computational novel AMR gene discovery [32]. The study is somewhat pioneer that employed CARD as surveillance algorithm for PGPR, it is however a novel study because all the twenty-two selected PGBR clearly showed the three AMR variants into perfect, strict and loose. This is an outright indication that the bacteria under study harbour resistance genes. The degree of prevalence of these genes varies among the PGBR. In consonance with earlier studies, drug efflux accounted for $67 \%$ as the mechanism of gene resistance with other mechanism. This study is in contrast with earlier studies leading to curation paradigm of CARD resistomes, which operates on four primary AMR gene family to, which resistance is conferred on specificity and sensitivity of antibiotics, which rest on the experimental interpretation of Minimum inhibitory concentration of the antibiotics. This study implicated LimA 23SrRNA methyltransferase in Paenibacillus Polymyxa and $P$. odorifer strain responsible for resistance to lincosamide antibiotics, while adeF genes are predictably responsible for resistance to fluoroquinolone and tetracycline antibiotics Delftia spp and Bradyrhizobium species.

The most variant genes (adeF, OXA-59, omp 38, amrA and amrB) of antibiotic resistance through antibiotic efflux pump. In the same vein, members of Rhizobium species use adeF genes for resistance to fluoroquinolone and tetracycline antibiotics family while Rhizobium species in this study use BcII, FosB genes for resistance to fosfomycin, cephalosporin, penam, phenicol antibiotics family $[27,28]$.

\section{Conclusion}

CARD as technological metagenomics tools here has further demonstrated its wider applicability as primarily better curation paradigm over ResFinder [31], ARGANNOT and even catalog of resistance alleles in NCBI [32]. The new CARD rules allow diversity inclusion of experimentally-proven data on axiomatic variation in agricultural or environmental isolates.

\section{References}

1.Singh JS. Plant growth promoting rhizobacteria. Resonance. 2013 Mar 1;18(3):275-281.

2. Jadhav HP, Shaikh SS, Sayyed RZ. Role of hydrolytic enzymes of rhizoflora in biocontrol of fungal phytopathogens: an overview. In: Mehnaaz S (ed) Rhizotrophs: plant growth promotion to bioremediation. Singapore:Springer; 2017.pp.83-203.

3. Glick BR. Bacteria with ACC deaminase can promote plant growth and help to feed the world. Microbiology Research. 2014;169: 30 9.

4. Geetha K, Rajithasri AB, Bhadraiah B. Isolation of Plant growth promoting rhizo bacteria from rhizosphere soils of green gram, biochemical characterization and screening for antifungal activity against pathogenic fungi. International Journal of Pharmaceutical Science Invention. 2014;3(9):47-54.

5. Glick BR, Cheng Z, Czarny J,Duan J. Promotion of plant growth by ACC deaminase-producing soil bacteria. European Journal of Plant Pathology. 2007;119: 329-39.

6. Glick BR. The enhancement of plant growth by free-living bacteria. Can J. Microbiol. 2005;41: 109-117.

7. Bloemberg GV, Lugtenberg BJJ. Molecular basis of plant growth promotion and biocontrol by rhizobacteria. Current Opinion Plant Biology. 2001;4:343-350.

8. Gwyn AB. Plant-Associated bacteria: survey, molecular phylogeny, genomics and recent advances. Springer(Dordrecht):InPlant-associated bacteria; 2006.pp.1-56. 
9. Ghodsalavi B, Ahmadzadeh M, Soleimani M, Madloo PR, Taghizad-Farid R. Isolation and characterization of rhizobacteria and their effects on root extracts of Valeriana officinalis. Australian Journal of Crop Science. 2013;7(3): 338-344.

10. Bloemberg GV, Lugtenberg BJ, Dekkers L. Molecular determinants of rhizosphere colonization by Pseudomonas. Ann Rev Phytopathol. 2001;38: 461-490.

11. Carmona-Hernandez S, Reyes-Pérez JJ, ChiquitoContreras RG, Rincon-Enriquez G, Cerdan-Cabrera CR, Hernandez-Montiel LG. Biocontrol of postharvest fruit fungal diseases by bacterial antagonists: A review. Agronomy. 2019 Mar;9(3):121.

12.Podile AR, Kishore GK. Plant growth-promoting rhizobacteria. In: Gnanamanickam SS(ed). Springer (Netherlands):Plant-Associated Bacteria; 2006:p.195230.

13. Bhardwaj D, Ansari M, Sahoo RK, Tuteja N. Bio fertilizers function as key player in sustainable agriculture by improving soil fertility, plant tolerance and crop productivity. Microbial. Cell Fact. (2014);13(66):pp. 1-10.

14. Camacho C, Coulouris G, Avagyan V, Ma N, Papadopoulos J, Bealer K, Madden TL. BLAST: architecture and applications. BMC Bioinformatics. 2009;10:421. doi:10.1186/1471-2105-10-421

15. Ahmad S, Tahir I, Shahri W. Effect of different storage treatments on physiology and postharvest performance in cut scapes of three Iris Species. J Agric Sci Technol. 2013; 15:323-331.

16. Droby S, Wisniewski M, Macarisinb D, Wilson C. Twenty years of postharvest biocontrol research: Is it time for a new paradigm? Postharvest Biology and Technology. 2009;52:137-145.

17. Kaplan D, Maymon M, Agapakis CM, Lee A, Wang A, Prigge BA. A survey of the microbial community in the rhizosphere of the dominant plant of the negev desert, Zygophyllumdumosum boiss using cultivation-dependent and independent methods. Am J Bot. 2013;100:17131725. doi: 10.3732/ajb.1200615.

18. Yashoda K, Manasa M, Vivek MN, Kekuda TP. Inhibitory effect of some plants of Western Ghats of Karnataka against Colletotrichum capsici. Science, Technology and Arts Research Journal. 2014 Aug 22;3(2):76-82.

19. Lema AA, Mudansiru A, Alexander BA, Sakinatu MJ. Evaluation of fungal species isolated from three different varieties of pepper (Capsicum chinense, C. frutescens and C. annum L.) in Dutsin-ma, Katsina State. Annals of Biological Sciences . 2018;6(1): 13-17.

20.Zankari E, Hasman H, Cosentino S, Vestergaard M, Rasmussen S, Lund O, Aarestrup FM, Larsen MV. Identification of acquired antimicrobial resistance genes. J Antimicrob Chemother. . 2012;67:2640 -2644.

21. McArthur AG, Waglechner N, Nizam F, Yan A, Azad MA, Baylay AJ, et al.. The comprehensive antibiotic resistance database. Antimicrob Agents Chemother. 2013;57(7):3348-57.

22. Baquero F, Tedim AS, Coque TM. Antibiotic resistance shaping multi-level population biology of bacteria. Frontiers in microbiology. 2013 Mar 6;4:15.
23. Gaby JC, Buckley DH. A comprehensive evaluation of PCR primers to amplify the nifH gene of nitrogenase. PloS one. 2012 Jul 25;7(7):e42149.

24.Federhen S. The NCBI taxonomy database. Nucleic Acids Res. 2012;40:D136 -D143.

25.Liu B, Pop M. ARDB-Antibiotic Resistance Genes Database. Nucleic Acids Res. 2009;37:D443-D447.

26. Eddy SR. Accelerated Profile HMM searches. PLoS Computational Biology. 2011;7:e1002195. doi:10.1371/journal.pcbi.1002195.

27. Figueiredo MV, Burity HA, Martínez CR, Chanway CP. Alleviation of drought stress in the common bean (Phaseolus vulgaris L.) by co-inoculation with Paenibacillus polymyxa and Rhizobium tropici. Applied soil ecology. 2008 Sep 1;40(1):182-8.

28. Koumoutsi A, Chen XH, Henne A, Liesegang $H$, Hitzeroth G, Franke P, Vater J, Borriss R. Structural and functional characterization of gene clusters directing nonribosomal synthesis of bioactive cyclic lipopeptides in Bacillus amyloliquefaciens strain FZB42. Journal of bacteriology. 2004 Feb 15;186(4):1084-96.

29. Jia B, Raphenya AR, Alcock B, Waglechner N, Guo P, Tsang KK, Lago BA, Dave BM, Pereira S, Sharma AN, Doshi S. CARD 2017: expansion and model-centric curation of the comprehensive antibiotic resistance database. Nucleic acids research. 2016 Oct 25:gkw1004.

30. Alcock BP, Raphenya AR, Lau TT, Tsang KK, Bouchard M, Edalatmand A, Huynh W, Nguyen AL, Cheng AA, Liu S, Min SY. CARD 2020: antibiotic resistome surveillance with the comprehensive antibiotic resistance database. Nucleic acids research. 2020 Jan 8;48(D1):D517-25.

31. Gupta SK, Padmanabhan BR, Diene SM, Lopez-Rojas R, Kempf M, Landraud L, Rolain JM. ARG-ANNOT, a new bioinformatic tool to discover antibiotic resistance genes in bacterial genomes. Antimicrobial agents and chemotherapy. 2014 Jan 1;58(1):212-20.

32.Zankari E, Hasman H, Cosentino S, Vestergaard M, Rasmussen S, Lund O, Aarestrup FM, Larsen MV. Identification of acquired antimicrobial resistance genes. Journal of antimicrobial chemotherapy. 2012 Nov 1;67(11):2640-4. 